\title{
Effectiveness of Quinacrine (Mepacrine) in Photosensitive Autoimmune Diseases, Lupus Erythematosus, and Dermatomyositis: Report on 38 Cases and Literature Review
}

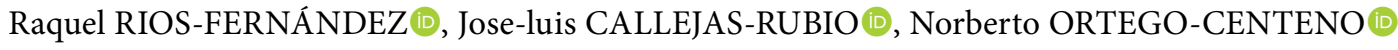 \\ Hospital San Cecilio, Autoimmune Diseases Unit, Granada, Spain
}

\begin{abstract}
Objectives: This study aims to report our experience with photosensitive autoimmune diseases including lupus erythematosus and dermatomyositis (DM) treated with quinacrine (Qn) as either monotherapy or combination with other antimalarials, steroids, and immunosuppressive therapy in an add-on regimen in light of a review of the relevant literature.

Patients and methods: The study included 38 patients ( 6 males, 32 females; mean age $45 \pm 8$ years; range, 23 to 72 years) with systemic lupus erythematosus (SLE), cutaneous lupus or DM who had been treated with Qn. The following data were obtained from the records of each patient: sex, age, diagnosis, duration of the disease, duration of treatment, smoking behavior, antimalarial treatment, concomitant treatment, and clinical indications, as well as Systemic Lupus Erythematosus Disease Activity Index (SLEDAI) and Cutaneous Lupus Erythematosus Disease Area and Severity Index (CLASI) activity before initiation, at the last visit, or when Qn treatment was completed. We carried out a MEDLINE search for previously reported cases that, together with our patients, served as the basis of this report.

Results: Of the 38 patients, 34 were suffering from SLE or cutaneous lupus and four from DM. Qn was dosed at 50 or 100 mg in most of the patients. Twenty-seven patients received Qn as an add-on regimen therapy. Clinical response was analyzed in patients with SLE or cutaneous lupus. Of the patients, 25 responded (68.4\%), $13(52 \%)$ had improved CLASI activity and $12(48 \%)$ had improved SLEDAl score. Fifty percent of the patients with DM responded. A total of 188 cases were identified from the literature. The most frequent diagnosis was cutaneous lupus (68.6\%), followed by SLE (32.6\%). Only $7.4 \%$ of the patients had DM. The majority of the patients received concomitant immunosuppressive medications. Treatment response was $73 \%$ in patients with SLE and/or cutaneous lupus and $35.7 \%$ in patients with DM. Side effects were scarce and the most frequent was yellow skin discoloration.

Conclusion: Quinacrine may be an alternative for patients with poor response or those who are intolerant to other antimalarials. Thus, Qn may aid in controlling the activity of photosensitive autoimmune diseases.

Keywords: Antimalarials; cutaneous lupus erythematosus; dermatomyositis; mepacrine; quinacrine; systemic lupus erythematosus.
\end{abstract}

For years, antimalarials have been used to treat several autoimmune diseases. ${ }^{1}$ The efficacy of antimalarials is related to a strong modulation of the immune response, ${ }^{2}$ as well as their photoprotective properties. ${ }^{3}$ Hydroxychloroquine (HCQ) and chloroquine (CQ) are often the first-choice therapy. Quinacrine $(\mathrm{Qn})$ is used as second-line therapy, particularly in patients with preexisting eye conditions for which treatment with HCQ or CQ is contraindicated, and in combination with other antimalarials in patients with treatment-resistance or only a partial response to CQ or HCQ. ${ }^{4}$ A 1,959 study showed the strength of Qn used with $\mathrm{HCQ},{ }^{5}$ and combination therapy has been used since. ${ }^{6}$

Received: March 28, 2018 Accepted: August 02, 2018 Published online: September 04, 2018

Correspondence: Raquel Rios-Fernández, MD. Hospital San Cecilio, Autoimmune Diseases Unit, 18016 Granada, Spain Tel: +34605392489 e-mail: rriosfer@hotmail.com 
In patients with lupus erythematosus (LE), combining low, medium, or high doses of glucocorticoids with another antimalarial drug (HCQ or CQ) or just with Qn seems to be a good choice for helping achieve better disease control; ${ }^{7}$ either combination therapy inhibits interferon alpha, something which is not possible when using glucocorticoids on their own. ${ }^{8}$ Furthermore, a synergistic/additive effect with cyclophosphamide, methotrexate, and cyclosporine- $\mathrm{A}$ has been described in patients with rheumatoid arthritis. ${ }^{9}$ Growing evidence has been presented over the last two decades of the anti-inflammatory, antithrombotic, anti-hyperlipidemic capacity of these agents, leading to a second renaissance and wider use. The usefulness of antimalarials is discussed in several reviews considering various diseases..$^{10,11}$ The photoprotective property of these agents is another interesting feature that may explain their beneficial effects in photosensitive autoimmune diseases. ${ }^{12}$ There are, however, still uncertainties about the real effectiveness of $\mathrm{Qn}$ and the most appropriate dosage. Therefore, in this study, we aimed to report our experience with photosensitive autoimmune diseases including LE and DM treated with Qn as either monotherapy or combination with other antimalarials, steroids, and immunosuppressive therapy in an add-on regimen in light of a review of the relevant literature.

\section{PATIENTS AND METHODS}

Data were retrospectively collected from 38 patients (6 males, 32 females; mean age $45 \pm 8$ years; range, 23 to 72 years) treated with Qn between March 2014 and June 2016 in the Autoimmune Diseases Unit of San Cecilio Hospital in Granada, Spain. The following data were obtained from the records of each patient: sex, age, diagnosis, duration of the disease, duration of treatment, smoking behavior, antimalarial treatment, concomitant treatment, and clinical indications, as well as Systemic Lupus Erythematosus Disease Activity Index (SLEDAI) and Cutaneous Lupus Erythematosus Disease Area and Severity Index (CLASI) activity before initiation, at the last visit, or when Qn treatment was completed. The patients were monitored every 12 weeks. At each visit, clinical data related to the patient's disease, side effects, and adherence to the indicated treatment were recorded. The study protocol was approved by the San Cecilio Hospital Ethics Committee. A written informed consent was obtained from each patient. The study was conducted in accordance with the principles of the Declaration of Helsinki.

We carried out a MEDLINE search for relevant articles, in any language, recorded between the inception of each database and December $30^{\text {th }}$ 2016, using the Medical Subject Headings (MESH) categories: antimalarials, Qn, cutaneous lupus erythematosus (CLE), systemic lupus erythematosus (SLE), and DM. All cases identified from the literature were included in the analysis. All article references were reviewed to identify additional studies that were not included in the electronic databases. The data from these cases, together with the information from our patients, served as the basis of this report.

The clinical diagnosis of SLE was based on the American College of Rheumatology criteria. ${ }^{13}$ The diagnosis of subacute cutaneous lupus erythematosus (SCLE) was defined according to Sontheimer, ${ }^{14}$ and discoid lupus (DL) was based on clinical and histological data. DM was diagnosed according to the criteria of Bohan and Peter. ${ }^{15}$

To analyze the treatment response, we compared the SLEDAI in SLE ${ }^{16}$ and the CLASI activity score $e^{17}$ for CLE obtained at the visit prior to initiation of therapy with the score obtained at the final visit. The SLEDAI is a clinical index for assessing lupus disease activity. It consists of 24 weighted clinical and laboratory variables of nine organ systems. The descriptor scores range from 1 to 8 , and the total possible score for all 24 descriptors is 105 .

The CLASI scoring system has been developed specifically for patients with CLE, taking into account both the anatomical region (e.g., face, chest, arms) and morphological aspects of skin lesions. Activity is scored on the basis of erythema, scale/hyperkeratosis, mucous membrane involvement, acute hair loss, and nonscarring alopecia. The total scores are calculated by simple addition based on the extent of the symptoms, which are documented according to the worst-affected lesion within a specific anatomical area. Treatment response in patients with DM was assessed by the physician based 


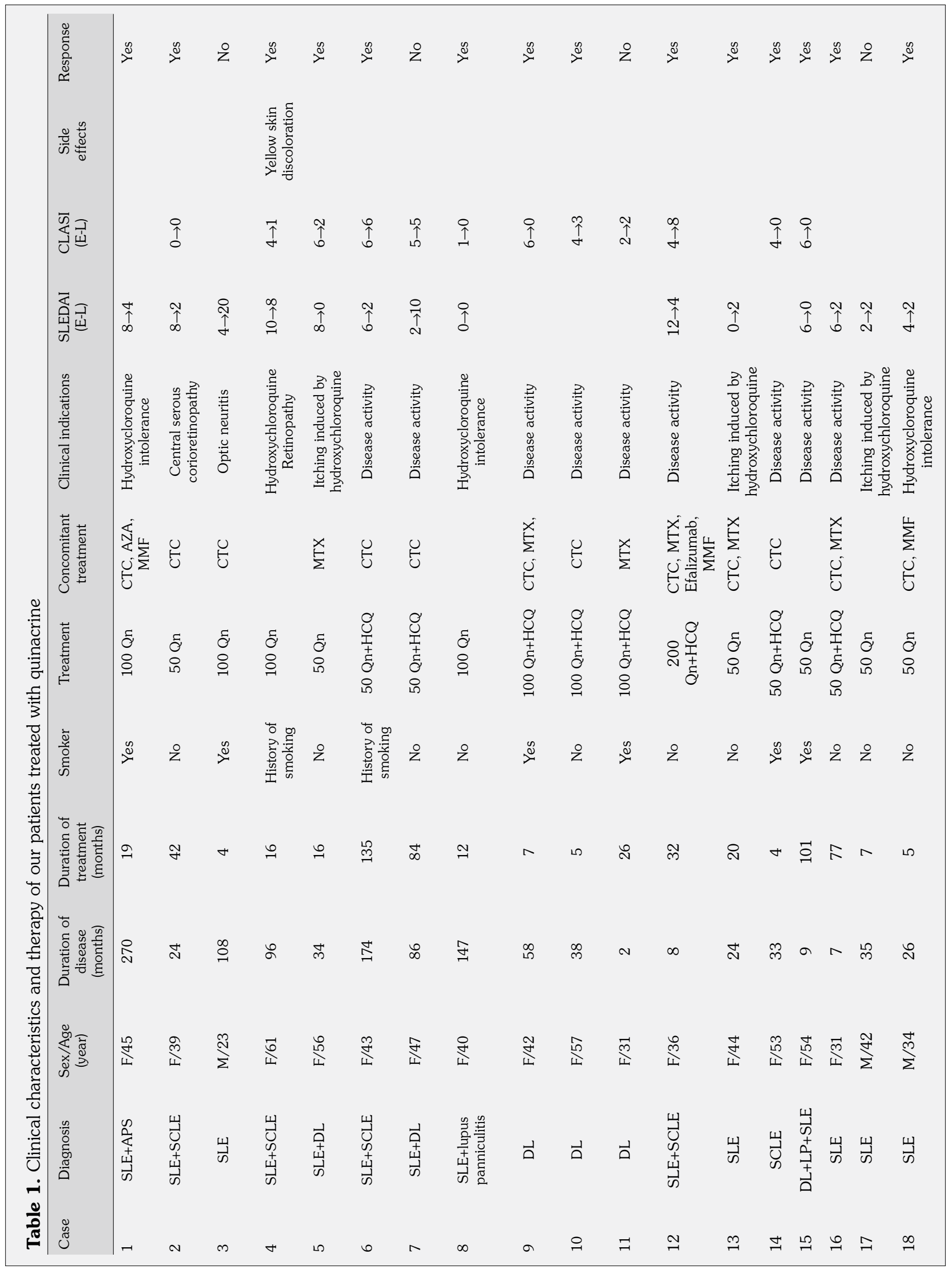




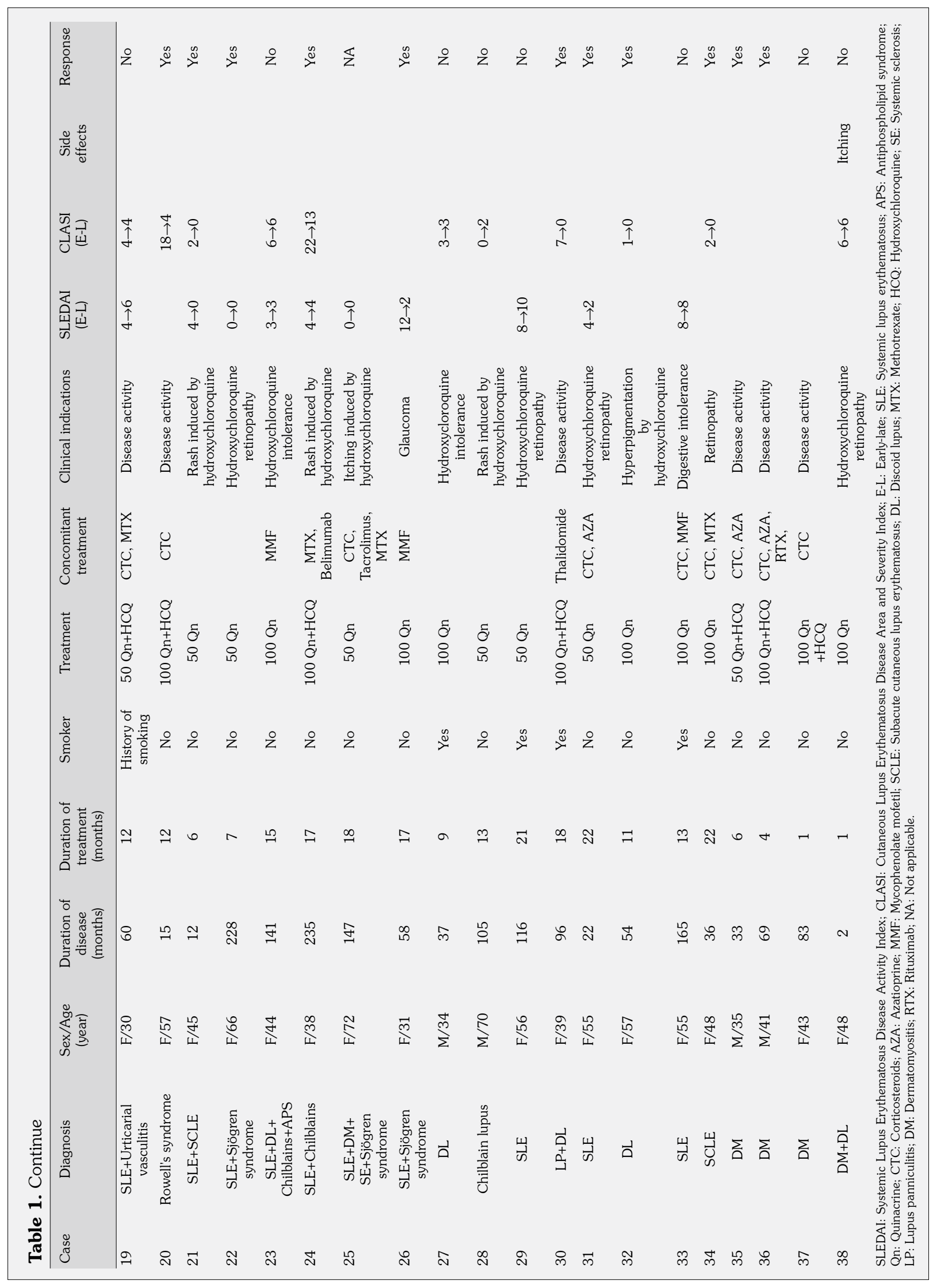


on the improvement in the extent of the disease (number of lesions, affected surface area, and activity of the disease), as expressed in erythema and pruritus. Qn was stopped in patients with unsatisfactory effects, or if a response was not deemed adequate.

Quinacrine was obtained from Normon laboratories (Tres Cantos, Madrid, Spain).
Off-label Qn use was requested for each patient. Qn was administered as an add-on regimen therapy in a minimal effective dose regimen if the patients had not responded to other antimalarial or corticosteroids and/or other immunosuppressors previously. If a patient had a different $\mathrm{Qn}$ dose regimen, the highest dose was considered for the study.

Table 2. Patient characteristics

\begin{tabular}{|c|c|c|c|c|c|c|}
\hline & \multicolumn{3}{|c|}{ Literature } & \multicolumn{3}{|c|}{ Present report } \\
\hline & $\mathrm{n}$ & $\%$ & Mean \pm SD & $\mathrm{n}$ & $\%$ & Mean \pm SD \\
\hline No of patients & 188 & & & 38 & & \\
\hline Age (year) & & & $41.4 \pm 12.8$ & & & $45.8 \pm 11.6$ \\
\hline Female & 129 & 68.6 & & 32 & 84.2 & \\
\hline $\begin{array}{l}\text { Diagnosis } \\
\text { Systemic lupus erythematosus } \\
\text { Discoid lupus } \\
\text { Subacute cutaneous lupus erythematosus } \\
\text { Acute lupus } \\
\text { Chilblain } \\
\text { Panniculitis (lupus profundus) } \\
\text { Chronic lupus } \\
\text { Skin lesions } \\
\text { Lupus tumidus } \\
\text { Hypertrophic lupus } \\
\text { Urticaria } \\
\text { Rowell } \\
\text { Dermatomyositis }\end{array}$ & $\begin{array}{c}60 \\
88 \\
31 \\
10 \\
4 \\
9 \\
5 \\
3 \\
19 \\
1 \\
0 \\
0 \\
14\end{array}$ & $\begin{array}{l}31.9 \\
46.8 \\
16.5 \\
5.3 \\
2.1 \\
4.8 \\
2.6 \\
1.6 \\
10.1 \\
0.5 \\
0 \\
0 \\
7.4\end{array}$ & & $\begin{array}{l}24 \\
11 \\
7 \\
0 \\
3 \\
3 \\
0 \\
0 \\
0 \\
0 \\
1 \\
1 \\
4\end{array}$ & $\begin{array}{c}63.2 \\
28.9 \\
18.4 \\
0 \\
7.9 \\
7.9 \\
0 \\
0 \\
0 \\
0 \\
2.6 \\
2.6 \\
10.5\end{array}$ & \\
\hline Disease duration in months & & & $120.1 \pm 100$ & & & $76.1 \pm 69.5$ \\
\hline Follow-up duration therapy in months & & & $30.3 \pm 32.7$ & & & $22.6 \pm 28.9$ \\
\hline $\begin{array}{l}\text { Quinacrine dosage (mg/day) } \\
50 \\
100 \\
150 \\
200 \\
300 \\
100-300\end{array}$ & $\begin{array}{c}5 \\
127 \\
1 \\
18 \\
12 \\
25\end{array}$ & $\begin{array}{c}2.6 \\
67.5 \\
0.5 \\
9.6 \\
6.4 \\
13.3\end{array}$ & & $\begin{array}{c}18 \\
19 \\
0 \\
1 \\
0 \\
0\end{array}$ & $\begin{array}{c}47.4 \\
10.3 \\
0 \\
2.6 \\
0 \\
0\end{array}$ & \\
\hline $\begin{array}{l}\text { Concomitant therapy with Qn } \\
\text { CTC } \\
\text { Hydroxychloroquine } \\
\text { Cloroquine } \\
\text { Methotrexate } \\
\text { Azatioprine } \\
\text { Mycophenolate mofetil } \\
\text { Thalidomide } \\
\text { Intravenous immunoglobulins } \\
\text { Rituximab } \\
\text { Tacrolimus } \\
\text { Belimumab } \\
\text { Efalizumab } \\
\text { Azatioprine, mycophenolate mofetil or cyclosporine }\end{array}$ & $\begin{array}{c}63 \\
108 \\
36 \\
14 \\
6 \\
1 \\
2 \\
1 \\
0 \\
0 \\
0 \\
0 \\
8\end{array}$ & $\begin{array}{c}33.5 \\
57.4 \\
19.1 \\
7.4 \\
3.2 \\
0.5 \\
1.1 \\
0.5 \\
0 \\
0 \\
0 \\
0 \\
4.3\end{array}$ & & $\begin{array}{c}21 \\
16 \\
0 \\
10 \\
4 \\
6 \\
1 \\
0 \\
1 \\
1 \\
1 \\
1 \\
0\end{array}$ & $\begin{array}{c}55.3 \\
42.1 \\
0 \\
26.3 \\
10.5 \\
15.8 \\
2.6 \\
0 \\
2.6 \\
2.6 \\
2.6 \\
2.6 \\
0\end{array}$ & \\
\hline $\begin{array}{l}\text { Response } \\
\text { Yes } \\
\text { No } \\
\text { Not applicable }\end{array}$ & $\begin{array}{c}132 \\
54 \\
2\end{array}$ & $\begin{array}{c}70.2 \\
28.7 \\
1.1\end{array}$ & & $\begin{array}{c}25 \\
11 \\
1\end{array}$ & $\begin{array}{c}68.4 \\
28.9 \\
2.6\end{array}$ & \\
\hline
\end{tabular}




\begin{tabular}{|lcc|}
\hline \multicolumn{2}{|c|}{ Table 3. Adverse events } & \\
\hline & $\begin{array}{c}\text { Literature } \\
(\mathrm{n}=188)\end{array}$ & $\begin{array}{c}\text { This study } \\
(\mathrm{n}=38)\end{array}$ \\
\hline Yellow skin discoloration & 26 & 1 \\
Drug rash & 3 & \\
Dermatitis & 2 & \\
Psoriasis & 1 & \\
Photosensitivity & 1 & \\
Itching & 1 & \\
Stroke & 1 & \\
Nausea & 2 & \\
Hepatitis & 2 & \\
Epigastralgia & 1 & \\
Fatigue & 1 & \\
Weight loss & 1 & \\
\hline
\end{tabular}

\section{RESULTS}

Patient demographics were provided in Table 1a. Of the 38 patients, 34 were suffering from SLE or cutaneous lupus. Of the 24 patients with SLE, Qn treatment was started due to skin disease in seven and disease activity in the others. Eleven patients had DL, seven had SCLE, three had chilblain lupus, three had lupus panniculitis, one had urticaria, and one had Rowell's syndrome. Only four patients had DM.

Quinacrine was dosed at 50 or $100 \mathrm{mg}$ in most of the patients and the mean follow-up was $22.6 \pm 28.9$ months. In our series, one patient received $200 \mathrm{mg} /$ day, 19 patients $100 \mathrm{mg} /$ day, and 18 patients $50 \mathrm{mg} /$ day. Qn was combined with HCQ in 16 patients. Twenty-seven patients had $\mathrm{Qn}$ as an add-on regimen therapy, most with steroids $(\mathrm{n}=21)$, followed by methotrexate in 10 patients, mycophenolate in six patients, and azathioprine in four patients.

The most frequent clinical indications were disease activity (36.8\%), followed by retinopathy (18.42\%), HCQ intolerance (itching, cutaneous rash or digestive intolerance) (15.8\%) and others.

Clinical response was analyzed in patients with SLE or cutaneous lupus. Responders were patients who improved their SLEDAI or CLASI activity. Twenty-five patients were responders (68.4\%), 13 (52\%) improved their CLASI activity and 12 (48\%) their SLEDAI. The median change in SLEDAI was $1.25 \pm 5.4$. No response (lack of treatment efficacy) was observed in four patients with SLE, two patients with DL, one patient with SLE and DL, one patient with SLE and urticarial vasculitis, and another patient with SLE, DL, and chilblains.

The indication for use of $\mathrm{Qn}$ in patients with DM was cutaneous disease, with $50 \%$ of patients responding. Altogether for lupus and DM, the response rate was 56\% with Qn alone and 73.3\% when Qn was combined with HCQ. In patients who received $50 \mathrm{mg} /$ day, the response rate was $72 \%$, with $100 \mathrm{mg} /$ day $63.2 \%$, and with $200 \mathrm{mg} /$ day $100 \%$ (single patient).

A total of 188 cases were identified in the literature (Table 1b), 6,7,11,18-25 of which 129 were females; the mean age was $41.4 \pm 12.8$ years (standard deviation) with a disease duration of $76.2 \pm 96.3$ months.

The most frequent diagnosis was cutaneous lupus (68.6\%), followed by SLE (32.6\%). Only $7.4 \%$ of patients had DM. Most patients received concomitant medication, predominantly steroids (33.5\%) and other types of antimalarials. Fourteen patients received methotrexate and six azathioprine. Most patients were treated using Qn with HCQ (55.3\%) or CQ (17.5\%).

The mean duration of therapy was $30.3 \pm 32.7$ months. A high percentage of patients responded well to the treatment (Table 2). Treatment response was $73 \%$ in patients with SLE or cutaneous lupus. No response was seen in two patients with SLE, 14 patients with DL, eight patients with tumid lupus (TL), four patients with SCLE, three patients with DL and SLE, one with SLE and acute cutaneous lupus (ACL), one with TL and DL, one with SLE and SCLE, one with ACL and lupus panniculitis (LP), and another with SLE and DL and TL. Cavazzana et al. $^{18}$ reported that SCLE lesions improved in $60 \%$ of cases, without a significant decrease in the activity. In addition, all acute lupus rashes and $50 \%$ of chilblains improved during therapy. In contrast, lupus panniculitis (lupus profundus) showed no amelioration.

In patients with $\mathrm{DM}$, the treatment response was $35.7 \%$ as indicated clinically by cutaneous symptoms. Most patients had more than one antimalarial as an add-on regimen. 
Altogether for lupus and DM, 85.1\% of the patients responded to $\mathrm{Qn}$ on its own, 68.3\% to Qn and HCQ, and 59.4\% to Qn and CQ. In four patients, a triple therapy was used (HCQ with Qn and $\mathrm{CQ}$ ), but only one responded to treatment. The most frequent dose administered was $100 \mathrm{mg}$. Among the patients who responded, treatment response with $\mathrm{Qn}$ alone or in combination was $91.67 \%, 84 \%, 83.3 \%, 68.5 \%, 100 \%$, and $80 \%$ for patients taking 300, 300-100, 200, 100, 150 and $50 \mathrm{mg} /$ day, respectively. Side effects were scarce; one patient had yellow skin discoloration while another experienced itching (Table 3).

\section{DISCUSSION}

Assuming that part of the antimalarial effect is due to the photoprotective effect, the aim of this study was to analyze the effectiveness of Qn, both alone or in combination with other antimalarials or immunosuppressive therapy, as an add-on regimen therapy for patients with photosensitive conditions, such as cutaneous lupus, SLE, and DM.

A high response rate to $Q n$ treatment was observed both among our patients and those reported in the reviewed literature $(68.4 \%$ and $70.2 \%$, respectively). The best response rate was observed in patients suffering from SLE or cutaneous lupus.

In cases of cutaneous lupus, the treatment may be used for patients with DL, SCLE, lupus panniculitis (lupus profundus), TL, hypertrophic lupus and chilblains. Although Cavazzana et al. ${ }^{18}$ reported that the therapy did not benefit patients with lupus profundus, we observed a good response in such patients; Feldmann et al. ${ }^{26}$ found that skin lesions improved significantly or cleared totally in all SCLE and half of chronic LE patients; and Lipsker et al. ${ }^{6}$ found that threequarters of DL, all SCLE, and all DL/SCLE patients demonstrated between 50\% and total clearance of lesions. As in most patients in the literature, we observed higher response in patients with DL and SCLE. We also noted the benefit of this therapy for urticaria and Rowell's syndrome. However, no response was observed in urticaria vasculitis sufferers.
In most of our patients with SLE, we observed improved SLEDAI score. In two cases (Cases 6 and 12), there was improved SLEDAI even though there was no change in CLASI score. However, Cases 17, 19 and 29 did not respond at all. Case 19 needed other immunosuppressive therapy while Cases 17 and 29 remained stable. Numerous systemic treatments were proposed for these patients, including mycophenolate mofetil, methotrexate, dapsone, thalidomide, intravenous immunoglobulin and biologicals; antimalarials were used as an add-on regimen. Similar to Toubi et al., ${ }^{7}$ we observed a corticosteroid-sparing effect with the combination therapy in this group of patients. Taken together, these studies provide evidence supporting the use of Qn for treating lupus patients.

The pathophysiology of photosensitive autoimmune skin reactions could be explained by ultraviolet (UV)-induced production of tumor necrosis factor alpha secretion that leads to keratinocyte apoptosis and the translocation of previously sequestered cellular antigens that then activate the immune system. ${ }^{27}$ Several explanations have been given for the effectiveness of antimalarials in inhibiting SLE activity, including lysosomal stabilization; inhibition of antigen presentation and cell-mediated cytotoxicity; inhibition of prostaglandin synthesis; inhibition of lipid peroxidation; inhibition of proinflammatory cytokine synthesis; influence on apoptosis, reduction of the levels of circulating immune complexes; antioxidant action, antithrombotic/ antiplatelet effects; $;$ and photoprotective effects. ${ }^{12}$ It has been shown that the accumulation of antimalarials in the skin exerts a local antiinflammatory effect after ultraviolet-B (UV-B) exposure, ${ }^{29}$ which could explain the benefit of this drug in conditions associated with photosensitivity. ${ }^{30}$

Antimalarials have also been used for treating cutaneous symptoms of DM since at least 1984 , after Woo et al. ${ }^{31}$ reported their successful use in this condition; however, the response rate was lower than that seen in patients with lupus. In our series, when considering the diseases individually, the responses were also worse in patients with DM compared with those in patients with lupus. To date, the response rates in this study are higher than those reported in the literature. ${ }^{11}$ DM patients, similar to those with LE, show 
significantly increased erythemal sensitivity to UV-B irradiation, ${ }^{27}$ while Qn seems less effective in treating the cutaneous symptoms of these patients. The high proportion of patients with DM that responded well in our series is probably due to the low number of patients. As in the previously published studies, most of our patients received glucocorticoids concomitantly with immunosuppressive agents.

In our patients, the best response was seen with the combination of Qn and HCQ. In most patients, this combination was not used due to intolerance or contraindication for the use of HCQ; if the association had been possible, it is likely that the response rate would have been higher. Qn can be an alternative treatment for patients who respond poorly or are intolerant to HCQ, although antimalarials, when combined, may exert a complementary effect, thus explaining the enhanced efficacy observed with the combination therapy. We did not treat our patients with the HCQ and CQ combination as it can increase ocular toxicity and is therefore not recommendable. Chang et al. ${ }^{25}$ reported improvement in $67 \%$ of their patients treated with a combination of HCQ and Qn, and 33\% of their cases treated with a combination of $\mathrm{CQ}$ and $\mathrm{Qn}$. The combined therapy could be effective not only in patients with photosensitivity, but also in those who are not suffering this condition. This type of therapy could induce remission in patients with long-lasting disease activity and severe systemic involvement of major organs; this may be associated with a significant corticosteroid-sparing effect.

With regard to dose, data from the literature indicate the use of $\mathrm{Qn}$ at between $300 \mathrm{mg} /$ day and $50 \mathrm{mg} /$ day. In the study of Rhodes and Allende, ${ }^{20}$ dosage concentration was from 100-300 mg/day; however, the number of patients treated with $100 \mathrm{mg} /$ day or $300 \mathrm{mg} /$ day was unclear.

In the study carried out by Carvazzana et al., ${ }^{18}$ treatment with $100 \mathrm{mg} /$ day and $50 \mathrm{mg} /$ day provided the same response rate. On the other hand, patients taking $100 \mathrm{mg} /$ day of Qn presented significantly faster improvement than those taking $50 \mathrm{mg} /$ day and a reduced steroid dosage. It would probably be advisable to start treatment at $100 \mathrm{mg} /$ day and continue with $50 \mathrm{mg} /$ day maintenance doses. It is, however, necessary to find the optimum dose for each patient, balancing the toxic effects and a clinically relevant response.

Yellow staining of the skin is a minor side effect of Qn use, although possible bone-marrow and/or nephrotoxic manifestations have been reported elsewhere. ${ }^{32}$ In both our patients and those collected from the literature, no serious side effects were observed during the follow-up period (of up to 30 years), with the exception of a case of severe hepatitis in a patient affected by SLE and chronic hepatitis $\mathrm{C}$ virus (HCV) infection. $^{18}$ The liver histology of this patient showed double damage, caused by aggressive hepatitis with piecemeal necrosis (due to chronic $\mathrm{HCV}$ hepatitis), and drug-induced-damage, represented by cholangiolitis and microinfarcts in portal spaces. Cavazzana et al. ${ }^{18}$ reported side effects in patients receiving $100 \mathrm{mg} /$ day, while none in patients receiving $50 \mathrm{mg} /$ day.

There are several limitations to our study. Firstly, this is not a clinical trial. Secondly, it includes patients with heterogeneous characteristics and a small sample size. However, this is a study with real-life conditions and it is striking that the responses observed in our patients are similar to those reported in the literature. We believe that this is an argument in favor of our results' consistency.

In conclusion, Qn (as an "off-label drug") can be useful in three clinical situations: (i) to avoid antimalarial ocular toxicity in patients with HCQ retinopathy or another ocular disease; (ii) to allow the use of antimalarials when other antimalarials are not tolerated (itching, rash due to HCQ, or digestive intolerance); and (iii) to improve a sub-optimal antimalarial response as an add-on therapy. Combining this therapy with other antimalarials can help control the activity of photosensitive autoimmune diseases, particularly SLE. Larger studies are necessary to evaluate the response to antimalarials and any differences across cutaneous subtypes. Further studies are also required to assess the optimal treatment regimen in different subsets, as well as treatment dosage and combinations.

\section{Declaration of conflicting interests}

The authors declared no conflicts of interest with respect to the authorship and/or publication of this article. 


\section{Funding}

The authors received no financial support for the research and/or authorship of this article.

\section{REFERENCES}

1. Ben-Zvi I, Kivity S, Langevitz P, Shoenfeld Y. Hydroxychloroquine: from malaria to autoimmunity. Clin Rev Allergy Immunol 2012;42:145-53.

2. Furst DE. Pharmacokinetics of hydroxychloroquine and chloroquine during treatment of rheumatic diseases. Lupus 1996;5:11-5.

3. Wozniacka A, Lesiak A, McCauliffe DP, SysaJedrzejowska A. Photoprotective properties of chloroquine phosphate. J Eur Acad Dermatol Venereol 2007;21:1133-4.

4. Wallace DJ. The use of quinacrine (Atabrine) in rheumatic diseases: a reexamination. Semin Arthritis Rheum 1989;18:282-96.

5. Tye MJ, White H, Appel B, Ansell HB. Lupus erythematosus treated with a combination of quinacrine, hydroxychloroquine and chloroquine. $\mathrm{N}$ Engl J Med 1959;260:63-6.

6. Lipsker D, Piette JC, Cacoub P, Godeau P, Frances C. Chloroquine-quinacrine association in resistant cutaneous lupus. Dermatology 1995;190:257-8.

7. Toubi E, Rosner I, Rozenbaum M, Kessel A, Golan TD. The benefit of combining hydroxychloroquine with quinacrine in the treatment of SLE patients. Lupus 2000;9:92-5.

8. Zhang X, Li Q, Fang L, Huang H, Hou C, Liao Z, et al.. Anti-Malarial Drugs (hydroxychloroquine and quinacrine) Decrease The Production Of InterferonAlfa Initiated By TLR-9 Agonist. 2013 ACR/ARHP Annual Meeting, October 25-30, 2013 in San Diego, CA, USA. Abstract number: 1613. 2013.

9. Langevitz P, Kaplinsky N, Ehrenfeld M, Pras M. Intractable RA--treatment with combined methotrexate, azathioprine and hydroxychloroquine. Br J Rheumatol 1989;28:271-2.

10. Rynes RI. Ophthalmologic considerations in using antimalarials in the United States. Lupus 1996;5:73-4.

11. Ang GC, Werth VP. Combination antimalarials in the treatment of cutaneous dermatomyositis: a retrospective study. Arch Dermatol 2005;141:855-9.

12. Dalmau J, Roé E, Corella F, García-Navarro X, Peramiquel L, Alomar A. Acute generalized skin eruption due to adalimumab: report of two cases. $\mathrm{J}$ Eur Acad Dermatol Venereol 2007;21:1105-6.

13. Tan EM, Cohen AS, Fries JF, Masi AT, McShane DJ, Rothfield NF, et al. The 1982 revised criteria for the classification of systemic lupus erythematosus. Arthritis Rheum 1982;25:1271-7.

14. Sontheimer RD. Subacute cutaneous lupus erythematosus: 25-year evolution of a prototypic subset (subphenotype) of lupus erythematosus defined by characteristic cutaneous, pathological, immunological, and genetic findings. Autoimmun Rev 2005;4:253-63.

15. Bohan A, Peter JB. Polymyositis and dermatomyositis (first of two parts). N Engl J Med 1975;292:344-7.

16. Bombardier C, Gladman DD, Urowitz MB, Caron $\mathrm{D}$, Chang $\mathrm{CH}$. Derivation of the SLEDAI. A disease activity index for lupus patients. The Committee on Prognosis Studies in SLE. Arthritis Rheum 1992;35:630-40.

17. Albrecht J, Taylor L, Berlin JA, Dulay S, Ang G, Fakharzadeh S, et al. The CLASI (Cutaneous Lupus Erythematosus Disease Area and Severity Index): an outcome instrument for cutaneous lupus erythematosus. J Invest Dermatol 2005;125:889-94.

18. Cavazzana I, Sala R, Bazzani C, Ceribelli A, Zane $\mathrm{C}$, Cattaneo R, et al. Treatment of lupus skin involvement with quinacrine and hydroxychloroquine. Lupus 2009;18:735-9.

19. Toubi E, Kessel A, Rosner I, Rozenbaum M, Lorber M, Paran D, et al. Quinacrine added to ongoing therapeutic regimens attenuates anticardiolipin antibody production in SLE. Lupus 2003;12:297-301.

20. Rhodes BL, Allende MF. Treatment of chronic discoid lupus erythematosus with quinacrine. Calif Med 1954;80:70-1.

21. Conner SK. Systemic lupus erythematosus; a report on twelve cases treated with quinacrine (atabrine) and chloroquine (aralen). Ann Rheum Dis 1957;16:76-81.

22. González-Sixto B, García-Doval I, Oliveira R, Posada C, García-Cruz MA, Cruces M. Quinacrine in the treatment of cutaneous lupus erythematosus: practical aspects and a case series. Actas Dermosifiliogr 2010;101:54-8.

23. Chung HS, Hann SK. Lupus panniculitis treated by a combination therapy of hydroxychloroquine and quinacrine. J Dermatol 1997;24:569-72.

24. Benoit S, Goebeler M. Mepacrine in recalcitrant cutaneous lupus erythematosus: Old-fashioned or Still Useful? Acta Derm Venereol 2015;95:596-9.

25. Chang AY, Piette EW, Foering KP, Tenhave TR, Okawa J, Werth VP. Response to antimalarial agents in cutaneous lupus erythematosus: a prospective analysis. Arch Dermatol 2011;147:1261-7.

26. Feldmann R, Salomon D, Saurat JH. The association of the two antimalarials chloroquine and quinacrine for treatment-resistant chronic and subacute cutaneous lupus erythematosus. Dermatology 1994;189:425-7.

27. Dourmishev L, Meffert $H$, Piazena $H$. Dermatomyositis: comparative studies of cutaneous photosensitivity in lupus erythematosus and normal subjects. Photodermatol Photoimmunol Photomed 2004;20:230-4.

28. Lenert P. Inhibitory oligodeoxynucleotides - therapeutic promise for systemic autoimmune diseases? Clin Exp Immunol 2005;140:1-10. 
29. Sjölin-Forsberg G, Berne B, Eggelte TA, KarlssonParra A. In situ localization of chloroquine and immunohistological studies in UVB-irradiated skin of photosensitive patients. Acta Derm Venereol 1995;75:228-31.

30. Wozniacka A, Carter A, McCauliffe DP. Antimalarials in cutaneous lupus erythematosus: mechanisms of therapeutic benefit. Lupus 2002;11:71-81.

31. Woo TY, Callen JP, Voorhees JJ, Bickers DR, Hanno R,
Hawkins C. Cutaneous lesions of dermatomyositis are improved by hydroxychloroquine. J Am Acad Dermatol 1984;10:592-600.

32. Imazawa T, Furukawa F, Yoshida J, Shinoda K, Imaida $\mathrm{K}$, Takahashi $\mathrm{M}$, et al. Ultrastructural localization of myelin bodies and acid phosphatase activities in the liver and kidney induced by quinacrine in rats. Eisei Shikenjo Hokoku 1990;108:66-70. 